\title{
Design and Implementation of a Combinatorial Optimization Multi-population Meta-heuristic for Solving Vehicle Routing Problems
}

\author{
Eneko Osaba and Fernando Díaz
}

Faculty of Engineering, University of Deusto, 48007, Bilbao, Spain

\begin{abstract}
This paper aims to give a presentation of the PhD defended by Eneko Osaba on November 16th, 2015, at the University of Deusto. The thesis can be placed in the field of artificial intelligence. Specifically, it is related with multipopulation meta-heuristics for solving vehicle routing problems. The dissertation was held in the main auditorium of the University, in a publicly open presentation. After the presentation, Eneko was awarded with the highest grade (cum laude). Additionally, Eneko obtained the PhD obtaining award granted by the Basque Government through.
\end{abstract}

Keywords - Meta-heuristics, Transportation, Vehicle Routing Problem, Traveling Salesman Problem, Golden Ball.

\section{INTRODUCTION}

$\mathrm{O}_{\mathrm{r}}$ November 16th, 2015, Eneko Osaba defended his PhD thesis related with multi-population meta-heuristics for solving vehicle routing problems, at the University of Deusto [1]. The dissertation was held in the main auditorium of the University. After the presentation, and the corresponding doubts and questions raised by the assessing committee, Eneko was awarded with the highest grade (cum laude).

The thesis was supervised by Dr. Fernando Diaz, associate professor in the University of Deusto. On the other hand, the assessing committee of the $\mathrm{PhD}$ dissertation was composed of Professor Mario Piattini (University of Castilla-La Mancha), Dr. Esther Alvarez (University of Deusto), and Dr. Xin-She Yang (University of Middlesex, London, UK).

The development of the thesis was funded by the Basque Government, thanks to a grant awarded through a competitive process by the education, language policy and culture department (Grant ID: BFI. 2011-56). It is also important to highlight that the thesis has the International Mention, and that Eneko obtained the PhD obtaining award granted by the Basque Government through a competitive process.

The main publications associated with the $\mathrm{PhD}$ thesis are [2-5]. The full text of the thesis, the main papers published, and the source code of the developed method are available on the personal webpage associated with the University of Deusto ${ }^{1}$.

\section{SuMmary OF THE PERFORMED WORK}

Transportation is an essential area in the nowadays society. There are different kinds of transportation systems, each one with its own characteristics. In the same way, various areas of knowledge can deal efficiently with the transport planning. Concretely, the thesis is focused

1 http://paginaspersonales.deusto.es/e.osaba in the area of artificial intelligence and vehicle routing problems.

The majority of the problems related with the transport and logistics have common characteristics. This means that they can be modeled as optimization problems, being able to see them as special cases of other generic problems. Much of the problems of this type have an exceptional complexity, requiring the employment of techniques for its treatment. There are different sorts of these methods. Specifically, the thesis centers its attention on meta-heuristics.

A great amount of meta-heuristics can be found it the literature. Anyway, due to the high complexity of the problems, there is no technique able to solve all these problems optimally. This fact makes the field of vehicle routing problems be a hot topic of research. For this reason, the thesis focuses its efforts on developing a new meta-heuristic to solve different kind of vehicle routing problems. The presented technique offers an added value compared to existing methods, either in relation to the performance, and the contribution of conceptual originality. Specifically, the designed meta-heuristic has been called Golden-Ball (GB), and it is based in soccer concepts [2, 3].

The main characteristics of the GB can be summarized as follows. The GB is a multiple-population based meta-heuristic. First, the whole population of solutions (called players) is randomly created. Then, these created players are randomly divided among a fixed number of subpopulations (called team). Each team has its own training method (or coach), which is randomly assigned in this first phase. This training is the way in which each player in the team individually evolves along the execution. Another important training is the called Custom Training. In these trainings, a player which is trapped in a local optimum receives a special training in cooperation with the best player of its team. Once this first phase is finished, the competition phase starts. This second step is divided in seasons, composed by weeks. All the teams train independently every week, and they face each other creating a competition league. At the end of every season, a transfer procedure takes place. In this procedure the players and coaches can switch teams. The competition phase is repeated until the termination criterion is reached.

With the aim of validating the proposed model, its obtained results were compared with the ones obtained by other four algorithms of similar philosophy. Additionally, four well-known routing problems were used in this experimentation. Additionally, in order to perform a fair and rigorous comparison, two different statistical tests were carried out: the Friedman's test and the Holm's test. Thanks to this experimentation it can be affirmed that the proposed method is competitive in terms of performance and originality.

Besides this, how the GB works with more complex problems has been also shown in the thesis. These complex problems have been directly extracted from real world situations. For this purpose, different transportation problems have been modeled and treated as 
complex routing problems. In this aspect, the contribution is not only the application of the GB to these problems. It should be added the formulation and the treatment of them, because it is the first time that such problems are addressed in the literature.

One of the most interesting real-world situations faced in the thesis is related to the newspaper distribution [5]. More specifically, the object of study was a medium-sized newspaper distribution company. The area of coverage of this company is at a provincial level, which means that it has to serve a set of customers distributed in separate towns and cities. The company has some principles, which are the base of their logistic planning. The first principle is to treat towns and cities as separate units. In this way, if one vehicle enters a city, or a town, it was forced to serve each and every customer located therein.

On the other hand, due to the current environmental requirements, the company has a simple but robust paper recycling policy. In this case, the objects to recycle are the newspapers not sold the previous day. Thus, as can be deduced, vehicles not only have to meet the delivery demands of the customers. Besides that, they have to collect at each point those newspapers that were not sold the day before.

In addition, the company takes into account certain factors in the routes planning process. The first one is related to the hours at which the deliveries and collections are done. The service is performed daily during morning from 6:00am to 15:00. Within this time window exists one range considered as peak hours. In this way, traveling costs from one point to another are greater if they are performed at peak hours.

\section{REFERENCES}

[1] E. Osaba. Design and implementation of a combinatorial optimization multi-population meta-heuristic for solving vehicle routing problems. $\mathrm{PhD}$ thesis, Deusto Institute of Technology University of Deusto, November 2015.

[2] E. Osaba, F. Diaz, R. Carballedo, E. Onieva, and A. Perallos. Focusing on the golden ball metaheuristic: An extended study on a wider set of problems. The Scientific World Journal, 2014.

[3] E. Osaba, F. Diaz, and E. Onieva. Golden ball: a novel metaheuristic to solve combinatorial optimization problems based on soccer concepts. Applied Intelligence, 41(1):145-166, 2014.

[4] E. Osaba, X.-S. Yang, F. Diaz, P. Lopez-Garcia, and R. Carballedo. An improved discrete bat algorithm for symmetric and asymmetric traveling salesman problems. Engineering Applications of Artificial Intelligence, 48:59-71, 2016.

[5] E. Osaba, X.-S. Yang, F. Diaz, E. Onieva, A. D. Masegosa, and A. Perallos. A discrete firefly algorithm to solve a rich vehicle routing problem modelling a newspaper distribution system with recycling policy. Soft Computing, pages 1-14, 2016.

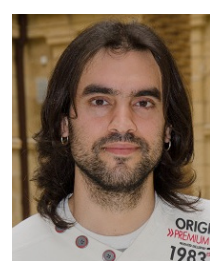

Eneko Osaba received the University degree and the Ph.D. degree in computer sciences from the University of Deusto, Bilbao, Spain, in 2010 and 2015, respectively. His doctoral thesis is focused on artificial intelligence, specifically in the field of combinatorial optimization, studying and developing heuristics and metaheuristics solving routing problems. At the same time, in September 2014, he began his career as a Lecturer, teaching statistics at the University of Deusto. He has participated in the development of more than 45 papers, published in different international scientific journals and conferences, being the first author in 29 of them. Among these papers, 21 of them have been published in international journals, having 15 of them JCR Impact factor (including 9 Q1). According to Scopus, he is the most prolific authors of the last 5 years under the search terms "Traveling Salesman Problem". He served as a member of the program committee of international conferences such as GECCO, HM and HAIS. Besides this, he is a regular reviewer in several scientific journals such as Soft Computing, Information Sciences, and Computers in Industry, and member of the editorial board of International Journal of Artificial Intelligence.

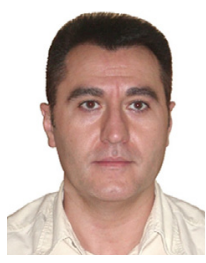

Fernando Diaz holds a BA in Computer Science and a $\mathrm{PhD}$ in Computer Science from the University of Deusto, and he is currently a lecturer of the Telecommunications Department at the Faculty of Engineering of Deusto University. From 1990 to 1992 he was an assistant researcher in the Artificial Intelligence Department of LABEIN research center (Bilbao, Spain). From 1992 to 1996 he was a PhD candidate in the Computing Languages and Systems Department at Deusto University, studying neural network algorithms for combinatorial optimization. His educational activity centers on the area of mathematics, teaching BA courses on Industrial Engineering, Computer Engineering, and Telecommunications Engineering. His main research interests include combinatorial optimization meta-heuristics applied to production scheduling and logistics. Other research interests include artificial neural networks and data mining. During the past few years he has carried out diverse studies in the areas of intelligent information retrieval, neural networks, genetic and evolving algorithms, and production scheduling and distribution, participating in various research projects funded by the European Commission, and the Basque Regional Government. $\mathrm{He}$ is also part of the Industrial Management and Logistics research team of the Engineering Faculty of Deusto University. 